\title{
Músculo esquelético: del nacimiento a la vejez, rutas hacia la falla mecánica y metabólica
}

\author{
Sofía Gitler ${ }^{1,2}$, Ibrahim Rámirez ${ }^{1}$, Ricardo Ramírez¹, Joel Medina ${ }^{1}$, Pavel Vázquez y Alicia Ortega ${ }^{1,3}$ \\ ${ }^{1}$ Departamento de Bioquímica, Facultad de Medicina, Universidad Nacional Autónoma de México; ${ }^{2}$ Facultad Mexicana de Medicina, Universidad \\ La Salle; ${ }^{3}$ Departamento de Bioquímica, Instituto Nacional de Perinatología, Ciudad de México, México
}

\begin{abstract}
Resumen
El músculo esquelético (ME) es el tejido más abundante y el mayor reservorio de proteínas del organismo. Transporta glucosa dependiente de insulina por los transportadores GLUT4 y mantiene la concentración de aminoácidos séricos. Por su masa y requerimientos energéticos, es fundamental para el equilibrio metabólico sistémico. En el presente trabajo presentamos el efecto de la desnutrición gestacional (DG) sobre las propiedades mecánicas y metabólicas del ME al nacimiento y en la vejez en un modelo animal. Los estudios mecánicos se realizaron en músculos aislados, y la determinación de GLUT4, transportadores de aminoácidos LAT2, SNAT2 y receptores de insulina (RI) se hizo en membranas aisladas de túbulos transversos (TT). La DG, en crías al nacimiento, resulta en disminución de la masa muscular, con aumento en la fuerza de contracción y resistencia a la fatiga, mientras que la expresión de RI, LAT2 y SNAT2 en los TT disminuye con el aumento en la expresión de GLUT4. En las ratas de 2 años (seniles) hay hipotrofia muscular y sarcopenia, la fuerza disminuye entre un 50 y un $70 \%$ en condiciones control y con DG, respectivamente, acompañándose de una menor expresión de LAT2, SNAT2 y RI en los TT. En conclusión, la DG afecta irreversiblemente al ME, efecto que podría ser similar en el ser humano, lo que nos ayudaría a entender los eventos que asocian a la DG con la debacle metabólica del ME y las enfermedades metabólicas de la vida adulta en humanos.
\end{abstract}

PALABRAS CLAVE: Músculo esquelético. Desnutrición gestacional. GLUT4. Vejez.

\begin{abstract}
Skeletal muscle (SM) is the most abundant tissue and the largest reservoir of protein in the body. It transports glucose in an insulin dependent manner by the glucose transporter type 4 (GLUT4) and contributes in the maintenance of serum amino acids concentration. By its mass and energetic requirements, it is fundamental for the systemic metabolic balance. In the present work, we present the effect of gestational undernourishment (GU) on the mechanical and metabolic properties of SM at birth and in old age in an animal model. Mechanical studies were performed on isolated muscles, while the GLUT4, amino acid transporters LAT2, SNAT2 and insulin receptors (IR) determination were performed on isolated transverse-tubule membranes (TT). The GU in offspring at birth, results in low muscle mass with increased contraction force and resistance to fatigue. However, in two-years old rats, there was muscle hypotrophy and sarcopenia, the force decreased between 50 and $70 \%$ in control rats and rats with GU respectively, accompanied by a lower expression of LAT2, SNAT2 and IR in TT. In conclusion, GU irreversibly affects the SM, an effect that could be similar in humans, which help us to understand the events that associate the GU with the metabolic debacle of SM and the metabolic diseases of human adulthood.
\end{abstract}

KEY WORDS: Skeletal muscle. Gestational undernourishment. Glucose transporter type 4. Aging.

Correspondencia:

Alicia Ortega

E-mail: aortega@unam.mx
Fecha de recepción: 07-08-2017

Fecha de aceptación: 07-08-2017

DOI://dx.doi.org/10.24875/GMM.17000008
Gac Med Mex. 2017;153;Sup 2:S60-S71

Contents available at PubMed

www.anmm.org.mx 


\section{Introducción}

Las alteraciones nutricionales son un problema que afecta a todos los grupos de edad. Sin embargo, la desnutrición durante el periodo gestacional tiene un efecto crítico sobre la diferenciación celular y el desarrollo embrionario. Condiciona graves alteraciones en las etapas posteriores, del nacimiento a la vejez. El músculo esquelético (ME) ejerce un papel crucial en el control metabólico. Es el tejido más abundante del cuerpo ( $50 \%$ del peso de un adulto promedio); es también el mayor reservorio de proteínas en el organismo ( $80 \%$ de su peso seco es proteína) y presenta un recambio importante de proteínas (12\% diario) ${ }^{1}$.

El ME es el resultado de un proceso de diferenciación altamente especializado que termina al nacimiento, lo mismo que la división celular ${ }^{2}$. Durante el proceso de diferenciación, la membrana plasmática del miotubo se invagina, dando lugar a una red de membranas celulares, los túbulos transversos (TT), que se extiende al interior de la fibra muscular en crecimiento para incrementar la superficie de contacto con el ambiente extracelular ${ }^{3,4}$. Los TT representan siete veces más superficie celular que la membrana plasmática (sarcolema) ${ }^{4}$.

Dos eventos están vinculados con el desarrollo de los TT: la transmisión del potencial de acción para iniciar el acople excitación-contracción, y con ello las propiedades mecánicas de la fibra muscular, y la expresión del transportador de glucosa GLUT4, isoforma que depende de la insulina para su inserción en las membranas de superficie y de esta manera transportar glucosa al interior de la fibra muscular. El GLUT4 es el mecanismo principal del ME para la captación de glucosa en la vida posnatal; la localización de los GLUT4 en los TT depende de la estimulación por la insulina, estando estos receptores mayoritariamente en los TT.

Por otra parte, la estimulación mecánica, el movimiento ${ }^{5,6}$, es el otro mecanismo que permite la expresión de GLUT4 en los TT sin la acción de la insulina. Se ha demostrado que GLUT4 es tres veces más abundante en los TT que en la sarcolema del ME adulto $^{7}$. En contraste, los receptores de insulina (RI) están distribuidos en iguales proporciones en las membranas de la sarcolema y la de los TT. EI ME, además de participar de manera fundamental en el mantenimiento de la concentración de glucosa sérica, también mantiene la concentración de aminoácidos séricos $^{8}$. Por su masa y requerimientos energéticos, el ME contribuye de manera esencial al equilibrio metabólico sistémico.

La fibra muscular contiene miles de núcleos que están unidos a la membrana celular por medio de proteínas que constituyen los filamentos intermedios ${ }^{9}$. Esta unión es fundamental para la mecanotransducción, mecanismo a partir del cual el movimiento, que se inicia con la activación de la membrana plasmática por el potencial de acción, se comunica mecánicamente al interior del núcleo dando inicio a la transcripción del ADN y a la síntesis de proteínas. El aumento de la masa muscular durante el crecimiento dependerá del transporte de glucosa y de aminoácidos, de la síntesis de proteínas y del movimiento.

Por otro lado, la hipotrofia muscular en la vejez ${ }^{10,11}$ $y$ en algunas distrofias musculares ${ }^{12,13}$ puede estar dada por una disminución en la participación de las células satélite, disminución en el aporte de nutrientes a la fibra muscular o un aumento en la proteólisis. La pérdida de la masa muscular cursa además con la muerte de las fibras musculares en un proceso conocido como sarcopenia ${ }^{14}$, siendo ambos factores condiciones incapacitantes en el envejecimiento.

En el presente trabajo estudiamos el efecto de la desnutrición gestacional sobre las propiedades mecánicas y la expresión del transportador de glucosa GLUT4, los RI, los transportadores de aminoácidos de cadena larga (LAT2) y los aminoácidos neutros de cadena corta (SNAT2) en los TT aislados de ME de ratas al nacimiento y en la vejez.

\section{Procedimientos experimentales}

\section{Animales}

El manejo de los animales se realizó según la Norma Oficial Mexicana NOM-062-ZOO-1999. Todos los animales empleados fueron manejados conforme a los lineamientos internacionales del uso de animales para la investigación. El estudio contó con aprobación del Comité de Ética de la Facultad de Medicina de la Universidad Nacional Autónoma de México.

\section{Apareamiento}

Se usaron ratas Wistar machos y hembras de $300 \mathrm{~g}$ que fueron colocadas en cajas con una sola hembra en un ambiente controlado con ciclos de luz/oscuridad de 12 horas a $25{ }^{\circ} \mathrm{C}$. Las fases del ciclo ovárico se determinaron por citología vaginal. El apareamiento fue confirmado por la presencia de esperm-atozoides 
en el frotis vaginal y datos clínicos de preñez, considerados indicadores del primer día de gestación. Posteriormente, un grupo de ratas preñadas iniciaron el protocolo de desnutrición.

\section{Protocolo de desnutrición gestacional}

A partir del primer día de gestación se estableció una dieta con un aporte calórico menor en un $30 \%$ con respecto a su control (12-15 g/día de alimento genérico para animales de laboratorio, Rodent Laboratory Chow, Agribrands Purina, México). El aporte de agua fue ad libitum durante los 21 días de la gestación. Se establecieron dos grupos de edad: al nacimiento (día cero) y a los 2 años (vejez) de edad, consideradas como ratas seniles ${ }^{15}$. Cada grupo tiene sus respectivos controles. Las ratas neonatas control (RN-C) y el grupo experimental, las ratas neonatas que provienen de la desnutrición gestacional (RN$D G)$. Las ratas adultas control corresponden a individuos de 3 meses de edad sin desnutrición (RA-C). Las ratas adultas control de 2 años con alimentación ad libitum toda su vida son consideradas ratas seniles control (RA-S control), las ratas seniles provenientes de la desnutrición gestacional con alimentación ad libitum toda su vida posnatal (RA-S con DG). Las ratas neonatas fueron medidas y pesadas inmediatamente después del nacimiento. Después fueron sacrificadas por decapitación. Durante el procedimiento se obtuvieron $300 \mathrm{ml}$ de sangre para la determinación de glucosa e insulina en plasma. Las crías con DG alimentadas ad libitum después del destete y que sobrevivieron hasta los 2 años de edad fueron sacrificadas por dislocación.

\section{Aislamiento de los músculos extensor largo de los dedos y sóleo para estudios mecánicos}

Se disecó el músculo total en las RN. Las RN se disecaron bajo el microscopio de luz y el músculo aislado se colocó en una cámara experimental de acrílico, en una solución que contiene, en mM, $135 \mathrm{NaCl}, 5 \mathrm{KCl}, 1 \mathrm{MgCl}_{2}, 2.5 \mathrm{CaCl}_{2}, 11$ dextrosa, 1 $\mathrm{Na}_{2} \mathrm{HPO}_{4}$ y $15 \mathrm{NaHCO}_{3}$, burbujeada con un $95 \%$ de $\mathrm{O}_{2}$ y un $5 \%$ de $\mathrm{CO}_{2}$, a pH 7.0. Se pesaron los músculos (peso húmedo) y posteriormente se colocaron en una cámara equipada con electrodos de platino para su estimulación eléctrica. El tendón distal del músculo extensor largo de los dedos (ELD) se sujetó a una pinza fija, y el tendón proximal a un transductor de fuerza. El transductor se conectó a un estimulador (GrassSD9) y este a un sistema de adquisición de datos. En las ratas de 2 años se disecaron los músculos ELD y sóleo bajo una lupa y se pesaron. El diseño experimental fue el mismo para ambos grupos de edad.

\section{Protocolo de estimulación}

La estimulación se realizó con pulsos eléctricos de 0.6 ms de duración hasta alcanzar el voltaje máximo para la respuesta máxima. Los músculos fueron estirados cuidadosamente hasta que se obtuviera la longitud óptima a la cual la tensión de contracción fuera la máxima, con el fin de estar en una longitud sarcomérica óptima (2.4 mm).

Se aplicó una estimulación tetánica de $75 \mathrm{~Hz}, 60$ $\checkmark$ por $3 \mathrm{~s}$, seguido por un periodo de descanso de 3 min. Esta fuerza será considerada el control para la tensión tetánica máxima. Al final del protocolo, el músculo se dejó reposar por 10 min antes de comenzar con el protocolo de fatiga.

\section{Protocolo del tren de estimulación para la fatiga}

La estimulación con pulsos eléctricos de $0.6 \mathrm{~ms}$ con $100 \mathrm{~V}$ se aplicó cada $0.3 \mathrm{~s}$ durante $20 \mathrm{~min}$. Cuando la tensión alcanzó el 30\% de la tensión original, el tren de estimulación se detuvo y se dio un periodo de descanso de $5 \mathrm{~min}$ antes del experimento de recuperación de tensión. Una estimulación tetánica de $75 \mathrm{~Hz}$ a $60 \mathrm{~V}$ por $1 \mathrm{~s}$ es seguida por un solo pulso de $1.5 \mathrm{~s}$ después de terminado el tétano; las estimulaciones consecutivas restauran la fuerza muscular. Este protocolo ha sido descrito previamente en otros trabajos $^{16,17}$.

\section{Aislamiento de las membranas de túbulos transversos y retículo sarcoplásmico}

Para el aislamiento de membranas se obtuvo el ME de grupos de $10 \mathrm{RN}$ (50-70 mg) siguiendo el procedimiento previamente descrito ${ }^{16}$. Brevemente, el músculo obtenido se homogenizó en una solución amortiguadora que contiene, en mM, 20 Tris-Malato y $100 \mathrm{KCl}$, a $\mathrm{pH} 7.0$, y se realizó una centrifugación diferencial y gradientes discontinuos de sacarosa $(43,35,27.5$ y $25 \%$ ), como ha sido descrito previamente ${ }^{16,18}$. La 
interfaz entre las fracciones 25 y $27.5 \%$ está enriquecida en la proteína caveolina-3 y el receptor de dihidropiridina (DHPR), que corresponden a marcadores de los TT. La fracción en la interfaz 27.5-35\% contiene la actividad de ATPasa de calcio (SERCA), por lo que corresponde al retículo sarcoplásmico.

\section{Actividad enzimática de la SERCA y la ATPasa de calcio de los túbulos transversos (PMCA)}

La actividad total de la ATPasa se midió mediante una determinación colorimétrica de fosfato inorgánico usando verde de malaquita, como ha sido descrito anteriormente ${ }^{19}$. Alícuotas de $5 \mathrm{mg} / \mathrm{ml}$ de proteína se incubaron en una solución que contiene, en $\mathrm{mM}, 100$ $\mathrm{KCl}, 5 \mathrm{MgCl}_{2}, 5 \mathrm{NaN}_{3}, 1$ ouabaína y 20 tris-malato, a pH 7 (ATPasa total) y en presencia de $1.0 \mathrm{mM}$ de EGTA para la PMCA. La reacción se detuvo con una solución que contenía $0.045 \%$ de verde de malaquita hidroclorada, $4.2 \%$ de molibdato de amonio en $4 \mathrm{~N}$ $\mathrm{HCl}, 0.8 \mathrm{ml}$ Triton-X 100 por cada $100 \mathrm{ml}$ de solución y $0.25 \mathrm{ml}$ de citrato de sodio (34\%), leyendo la absorbancia a $660 \mathrm{~nm}$.

\section{Determinación de la glucosa en sangre}

Se tomó aleatoriamente una gota de sangre para cuantificar las concentraciones séricas de glucosa, usando un glucómetro Accu Check Active ${ }^{\mathrm{TM}}$ (Roche, lote de tiras reactivas número 380 ).

\section{Determinación de la insulina en sangre}

Se obtuvieron $0.5 \mathrm{ml}$ de sangre sin anticoagulante. El suero se separó por centrifugación inmediatamente después de la decapitación. Las concentraciones de insulina se determinaron por ensayo de micro-ELISA usando un procedimiento estandarizado que se basa en el kit de insulina DSL-10-1600 Active $^{\text {TM }}$ (Diagnostic Systems Laboratories, Inc., Webster, TX, EE.UU.) y el equipo lector de ELISA BIO-RAD modelo 3550.

\section{Inmunoblot}

Una muestra de TT, $50 \mathrm{mg} / \mathrm{ml}$ de proteína, se desnaturalizó y colocó en geles SDS-PAGE al 10\%. Después, las proteínas fueron transferidas a una membrana de nitrocelulosa (Bio Rad) durante $1 \mathrm{~h} \mathrm{(100} \mathrm{V,}$
4C). La membrana se incubó durante $1 \mathrm{~h}$ con los siguientes anticuerpos: DHPR (ab2862), DHP (CACNA1S), caveolina-3 (Santa Cruz Biotechnology, 1:600), GLUT-4 (SC3566), LAT2 (SC27581 LAT2), SNAT2 (SC166366 SNAT2) y RI subunidad beta (3025s receptor beta de insulina). La determinación cuasicuantitativa se hizo por densitometría. Los resultados se expresaron en unidades de densidad normalizada (UDN) \pm la desviación estándar del promedio.

\section{Resultados}

La disminución del $30 \%$ en los requerimientos calóricos de la ingesta durante la gestación resultó en un aumento del $40 \%$ en la mortalidad de las crías, que no fueron consideradas en el presente estudio.

La figura 1 A nuestra una fotografía de las ratas neonatas: control (RN-C) y con desnutrición gestacional (RN-DG) al día 0, como muestra de sus proporciones corporales. La figura 1B muestra el peso corporal promedio de las RN-C, de $6.8 \pm 0.5 \mathrm{~g}$, y de las RN-DG, de $4.1 \pm 0.6 \mathrm{~g}$, un $34 \%$ menos peso corporal. El peso

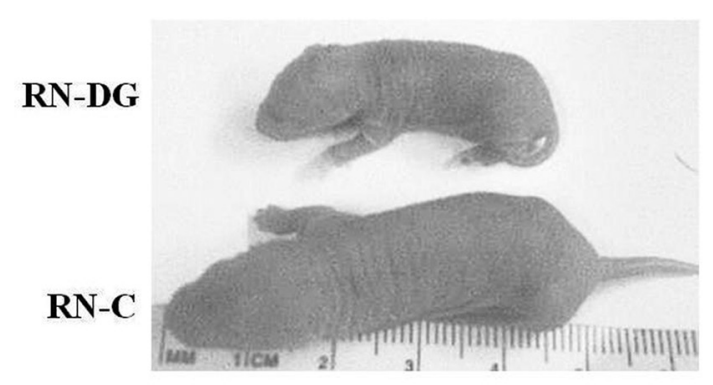

A
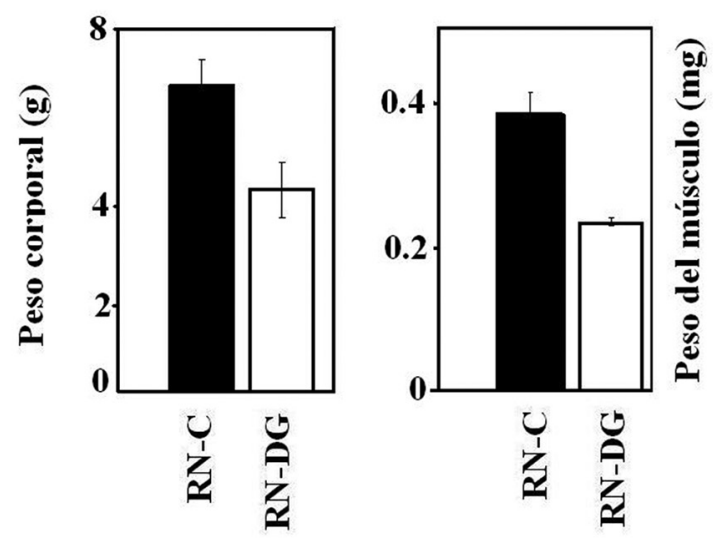

Figura 1. A: dimensiones de la rata neonata control y con desnutrición gestacional al día 0. B: peso corporal promedio y peso promedio del músculo esquelético en ratas neonatas al nacimiento (día 0), control $(R N-C)$ y con desnutrición gestacional (RN-DG). 
promedio del músculo (húmedo) que comprende los músculos dorsales y los de las extremidades posteriores, expresado por animal, fue de $0.37 \pm 0.004 \mathrm{~g}$ para las RN-C y de $0.21 \pm 0.001 \mathrm{~g}$ para las RN-DG, un $37 \%$ menos peso en masa muscular.

\section{Propiedades mecánicas del músculo esquelético neonatal}

Para evaluar la función muscular estudiamos las propiedades mecánicas de los músculos ELD aislados en las RN. En la figura 2 observamos que la fuerza de contracción y la resistencia a la fatiga aumentan en los músculos de las RN-DG, como ha sido reportado previamente ${ }^{16}$. La fuerza incrementa un $20 \%$ y la resistencia a la fatiga aumentó el $10 \%$. La potencia muscular es un $24 \%$ mayor en el músculo de las ratas RN-DG.

\section{Propiedades mecánicas del músculo esquelético en la vejez}

Para evaluar la función muscular de las ratas en la vejez estudiamos las propiedades mecánicas de los músculos aislados, ELD y sóleo, como representativos del músculo rápido o glucolítico y del músculo lento $u$ oxidativo, respectivamente. La figura $3 \mathrm{~A}$ muestra un registro representativo de la estimulación tetánica del músculo ELD en la rata adulta joven (RA-J). La figura $3 \mathrm{~B}$ muestra un registro representativo de la estimulación tetánica del músculo ELD en la rata adulta senil (RA-S). La fuerza de contracción y la resistencia a la fatiga durante el tétanos del músculo ELD disminuyen en un $50 \%$ en la RA-S. Por el contrario, en el músculo lento la vejez tiene poco efecto sobre el desarrollo de fuerza. La figura $3 \mathrm{C}$ muestra un registro representativo de la estimulación tetánica del músculo sóleo en la RA-J. La figura $3 \mathrm{D}$ muestra un registro representativo de la estimulación tetánica del músculo sóleo en la RA-S.

Aunque la fatiga en la estimulación tetánica es más intensa en el músculo senil, aquella que se obtiene a partir de un tren de estimulación es similar en la RA-J y en la RA-S. La recuperación de la fuerza de contracción después de la fatiga es de un $20 \%$ de la fuerza inicial en el músculo ELD de la RA-S, comparada con el $100 \%$ de recuperación de la RA-J. El efecto de la vejez sobre el músculo rápido es importante, disminuyendo la fuerza de contracción y la recuperación de la fatiga. Sin embargo, en el músculo lento no ejerce un efecto importante sobre la fuerza de contracción ni en la fatiga.

\section{Área transversal del extensor largo de los dedos y número de fibras musculares en el músculo joven y senil}

El peso promedio de los músculos de las ratas de 2 años fue, para las ratas control RA-S, de $700 \pm 23 \mathrm{~g}$, y en las RA-S con DG fue de $675 \pm 35 \mathrm{~g}$.

En términos generales, el área muscular (hipotrofia) de los músculos en la vejez disminuye, al igual que el número de células (sarcopenia) ${ }^{14,20}$. La figura $4 \mathrm{~A}$ muestra la relación del área transversal del músculo ELD y el número de fibras del ELD en función de la edad. La hipotrofia se inicia antes que la sarcopenia. Al año de edad hay una disminución del 5\% en el área transversal del ELD y no hay disminución en el número de fibras musculares en comparación con la RA-J. Para los 2 años de edad, el área transversal
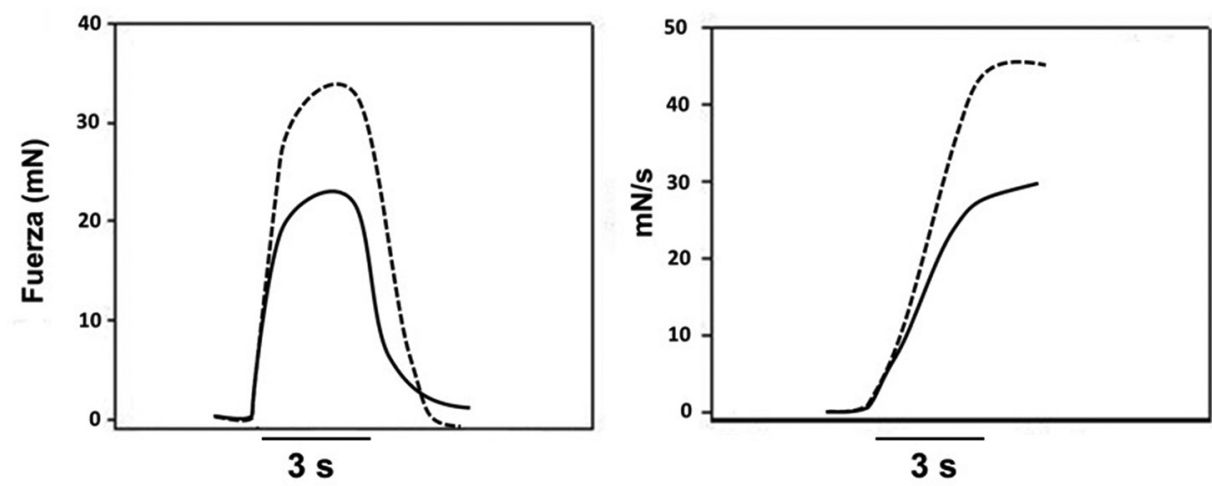

\section{A}

Tiempo (s) ${ }_{\mathrm{B}}$

Figura 2. Fuerza de contracción en el músculo extensor largo de los dedos (estimulación tetánica $75 \mathrm{~Hz}, 100 \mathrm{~V}, 3 \mathrm{~s}$ ). A: representa un tétanos de la rata $R N-C$ (línea continua) y de la RN-DG (línea punteada). B: corresponde a la integral de la fuerza (potencia muscular). Líneas continuas: rata $R N-C)$; líneas punteadas: $R N-D G$. 

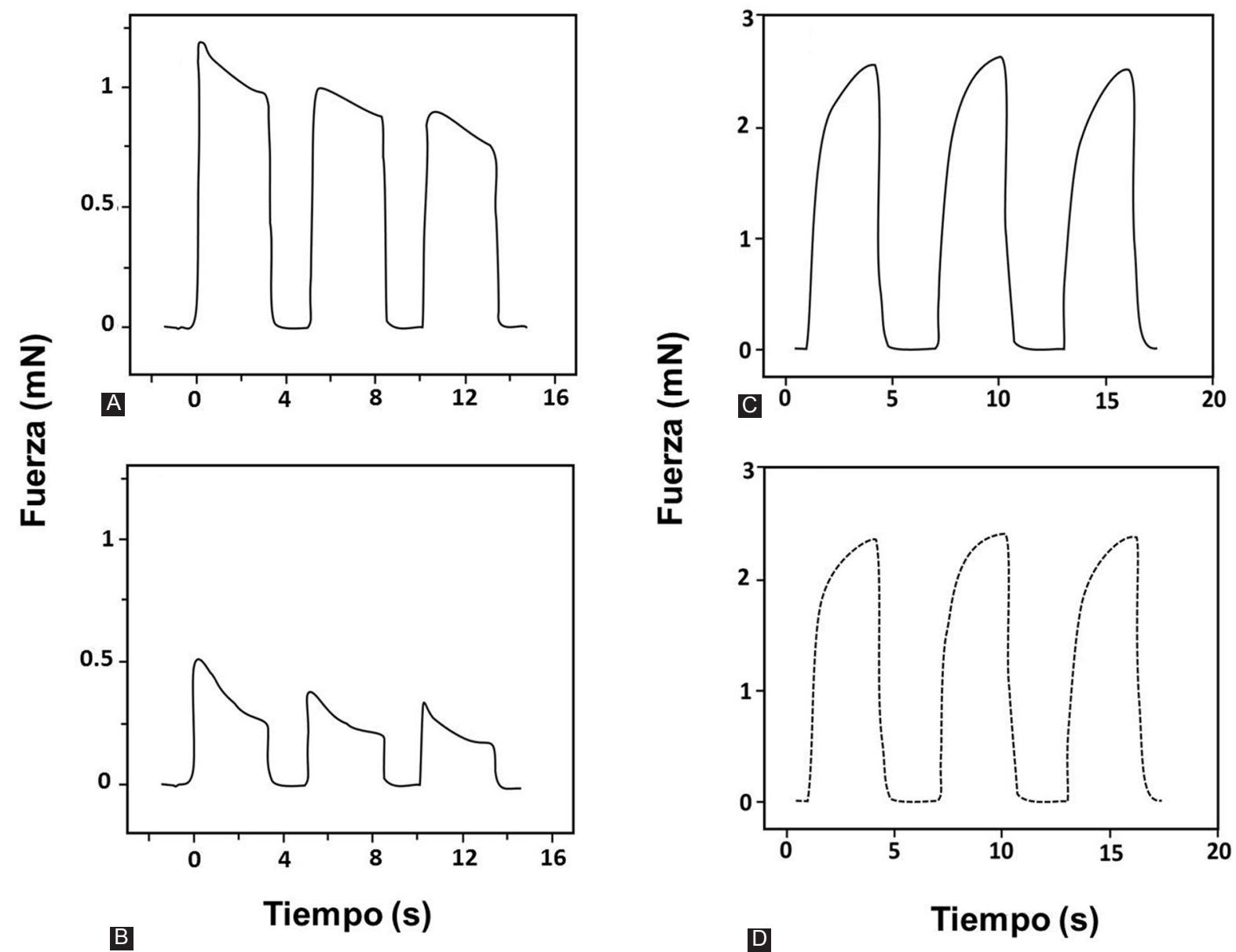

Figura 3. Fuerza de contracción y fatiga en la estimulación tetánica ( $75 \mathrm{~Hz}, 100 \mathrm{~V}$, 3s). Tres tétanos consecutivos en el músculo extensor largo de los dedos. A: RA-J. B: RA-S. Tres tétanos consecutivos en el músculo sóleo. C: RA-J. D: RA-S.

disminuye un $17 \%$ y el número de fibras disminuye un $25 \%$. La figura 4 B corresponde a la pérdida de la fuerza de contracción en función de la edad en las RA-S control y en las RA-S con DG. En las RA-S con $D G$, la pérdida de la fuerza de contracción es más intensa y disminuye de manera lineal. Sin embargo, a los 18 meses de edad ambos grupos tienen una pérdida simular de fuerza. La figura $4 \mathrm{C}$ muestra una fotografía de la región del músculo tibial anterior de una RA-S con DG de 2 años. Existe una importante sustitución del tejido muscular por tejido conectivo (fibras de colágena y adiposidad).

\section{Determinación de la actividad de la SERCA y PMCA en membranas aisladas}

Para determinar el efecto de la edad sobre la relajación muscular, evento dependiente de la energía del ATP y de las concentraciones de calcio intracelular, determinamos la actividad hidrolítica de la SERCA en membranas aisladas de RS del músculo de las RA-J y las RA-S. La determinación de la actividad de SERCA y PMCA en las ratas RN-C y RN-DG ha sido reportada previamente ${ }^{16}$. La figura 5 A muestra la actividad de la SERCA en las RA-J, las RA-S control y las RA-S con DG. La actividad disminuyó un $40 \%$ a los 30 min del inicio de la reacción en las RA-S en comparación con las RA-J. En las RA-S con DG, la actividad de la SERCA disminuyó un $45 \%$ adicional a la pérdida observada solo por el envejecimiento. El envejecimiento y la desnutrición durante la gestación tienen un efecto deletéreo sinérgico en el $\mathrm{ME}$ rápido.

Adicional a la función de la SERCA para regular las concentraciones de calcio citosólico e iniciar la relajación del músculo, se encuentra la actividad de la PMCA. La figura $5 \mathrm{~B}$ muestra la actividad de la PMCA en los TT de las RA-J, las RA-S control y las RA-S con DG. La actividad de la PMCA disminuyó un $85 \%$ a los 30 min del inicio de la reacción en las RA-S en 
comparación con las RA-J. Solo un 5\% adicional de la actividad de PMCA disminuyó en las RA-S con DG.

En la vejez, ambos mecanismos reguladores de calcio, tanto en el RS como en los TT, disminuyen de manera importante su actividad y se ven más afectados si el sujeto estuvo en DG.

La disminución de la actividad de las proteínas reguladoras del calcio, la pérdida de la fuerza de contracción, la hipotrofia y la sarcopenia son efecto de la vejez. La fuente principal de energía del ME es la glucosa, especialmente para el ME rápido, el tipo de músculo más afectado en sus propiedades mecánicas.

\section{Determinación de los transportadores de glucosa GLUT4 y los receptores de insulina en los túbulos transversos}

A partir de los músculos rápidos obtenidos de las ratas neonatas se aislaron las membranas de los TT. En el caso de las RA-S y las RA-J, se utilizaron solamente los músculos ELD para el aislamiento de los TT. La figura 6 muestra la imagen representativa para la determinación de GLUT4 en los TT. La figura $6 \mathrm{~A}$ es un inmunoblot con anti-GLUT4 en las RN-C y las RN-DG, en el que se observa un aumento del doble en la expresión de los GLUT4 en las RN-DG en comparación con las $\mathrm{RN}-\mathrm{C}$, como fue reportado previamente ${ }^{16}$.

En las ratas seniles, la expresión de GLUT4 aumentó en un $25 \%$ en las RA-S con DG comparadas con las RA-S control. Aunque en menor proporción, la expresión de GLUT4 en las RA-S con DG también aumentó a consecuencia de la DG. La figura $5 \mathrm{~B}$ muestra el análisis estadístico expresado en UDN $(n=3)$. La normalización se hizo a partir de las unidades de densidad obtenida de sus respectivos controles.

La inserción de GLUT4 en los TT responde esencialmente a dos estímulos: la presencia de insulina y su interacción con los RI presentes
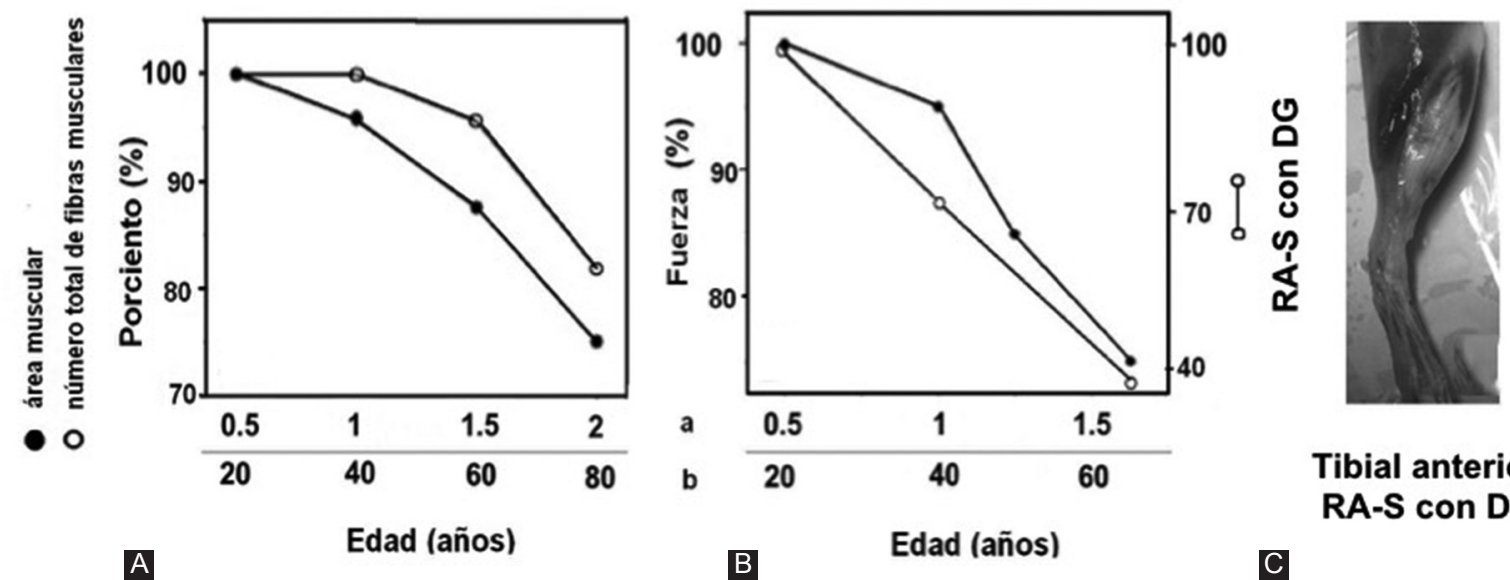

Tibial anterior RA-S con DG

Figura 4. A: hipotrofia (•) y sarcopenia (O) en función del envejecimiento. B: fuerza de contracción en función del envejecimiento en RA-S control (•) y RA-S con DG (O). C: ejemplo del músculo tibial anterior de una RA-S con DG, mostrando el músculo.
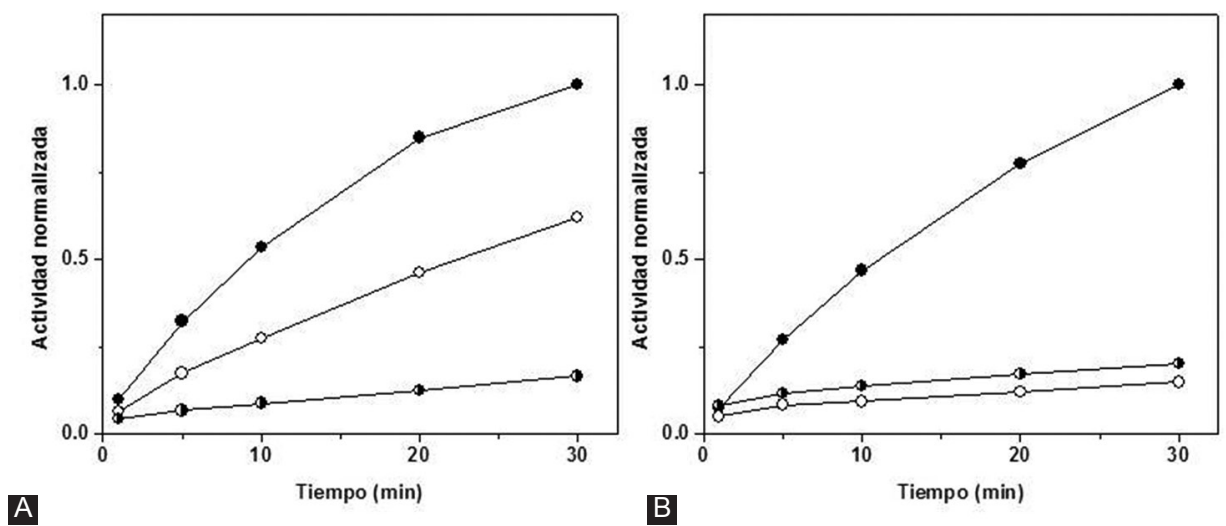

Figura 5. A: actividad hidrolítica de la SERCA. B: actividad hidrolítica de la PMCA. Retículo sarcoplásmico y túbulos transversos aislados de músculo esquelético rápido. Ratas $R A-J(\bullet), R A-S$ control (O) y RA-S con DG (⿶). 


\section{anti-GLUT4}
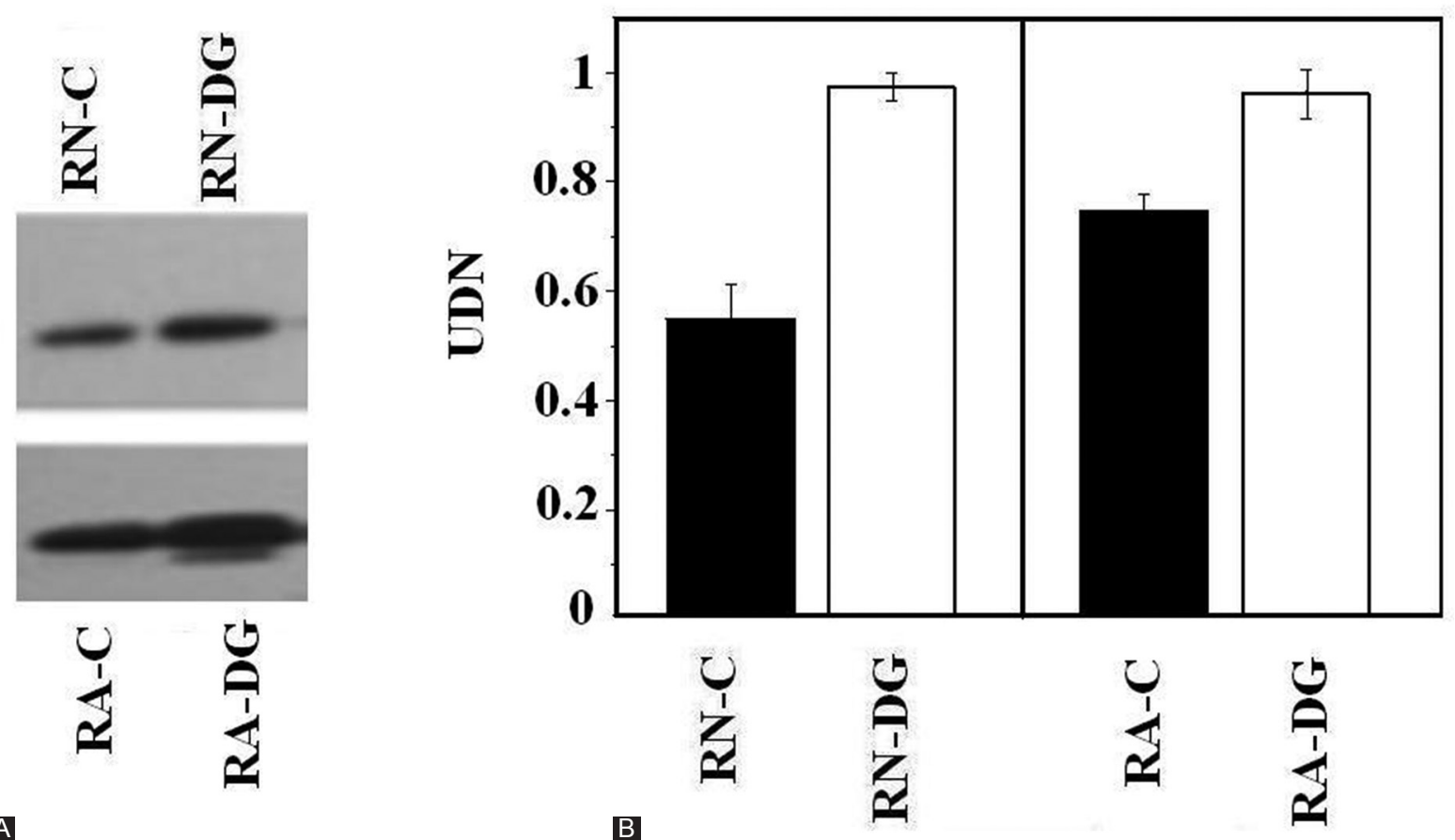

A

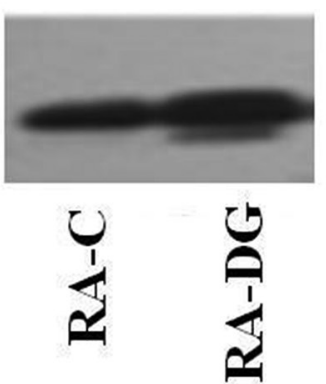

Figura 6. Western blot con anti-GLUT4 en los túbulos transversos (TT) aislados de músculo de ratas RN-C, RN-DG, RA-S control y RA-S con $D G$. A: las bandas corresponden a la misma cantidad de $T T$ (mg proteína). B: corresponde al promedio $(n=3)$ expresado en UDN $\pm D E$. La normalización se realizó respecto a los valores de UDN máxima.

principalmente en los TT, y como efecto del movimiento durante la contracción de las fibras musculares. La presencia aumentada de GLUT4 en los animales con DG, tanto al día 0 como en la senectud, plantea determinar la expresión de RI en TT aislados. La figura 7 A muestra una imagen representativa del Western Blot con anti-RI, que demuestra la presencia de estos receptores en los TT de las RN-C, las RN$D G$ y las RA-S control y RA-S con DG. La figura $7 \mathrm{~B}$ es el análisis estadístico en UDN $(n=3)$. La expresión de Rl fue un $43 \%$ menor en las RN-DG que en las RN-C. Las RA-S con DG expresaron un $18 \%$ menos RI en comparación con las RA-S control. El efecto de la $D G$ sobre la disminución del RI es más importante en las RN; sin embargo, el efecto se conserva hasta la vejez.

El ME es el reservorio de proteínas de los vertebrados. La síntesis de proteínas depende, entre otros factores, del aporte de glucosa y del transporte de aminoácidos, por lo que, en condiciones de hipotrofia en la desnutrición y la vejez, es importante determinar la expresión de los transportadores de aminoácidos en los TT.

\section{Determinación de los transportadores de aminoácidos en los túbulos transversos}

El músculo de animales con DG es menor, aunque la relación músculo/peso corporal se conserva. Se ha demostrado que el área de los TT está disminuida en las ratas $\mathrm{RN}-\mathrm{DG}^{16}$. La presencia de transportadores de aminoácidos de cadena larga (LAT2) y de aminoácidos pequeños neutros (SNAT2) es especialmente importante en el ME del recién nacido ${ }^{21}$. La figura 8 A muestra la imagen representativa del Western Blot para anti-LAT2 en RN-C, RN-DG, RA-S control y RA-S con DG. La figura $8 \mathrm{~B}$ representa el análisis estadístico $(n=3)$ expresado como el promedio de las UDN. La expresión de LAT2 en las RN-DG fue un $70 \%$ menor que en las RN-C. En las RA-S con DG, la expresión de LAT2 en los TT disminuyó también un $70 \%$ respecto a las RA-S control. Estos resultados indican que el transporte de aminoácidos de cadena larga en el ME es muy importante al nacimiento y disminuye de manera similar en ambas condiciones: la desnutrición gestacional y la vejez. 


\section{anti-RI}

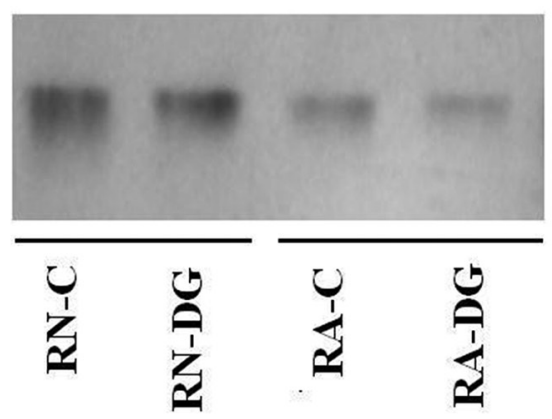

A

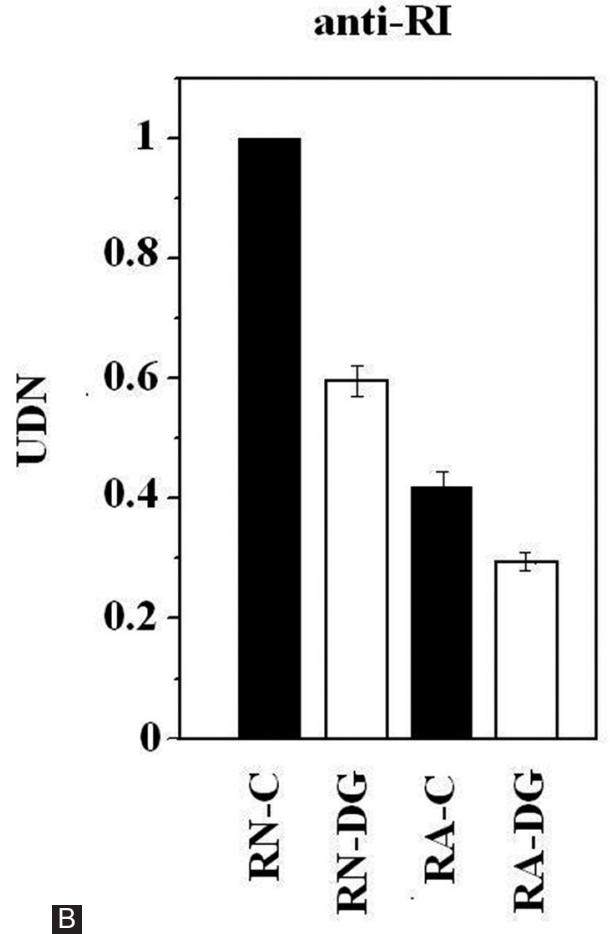

B

Figura 7. Western Blot con anti-Receptor de Insulina (subunidad beta) en los TTs aislados de músculo de ratas RN-C, RN-DG, RA-C y RA-DG; A: Las bandas corresponden a la misma cantidad en mg/proteína de TTs. B: corresponde al promedio de las UDN $(n=3) D E$. La normalización se realizó en relación a los valores de UDN máxima.

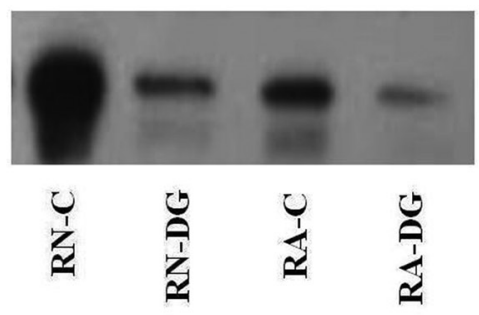

A

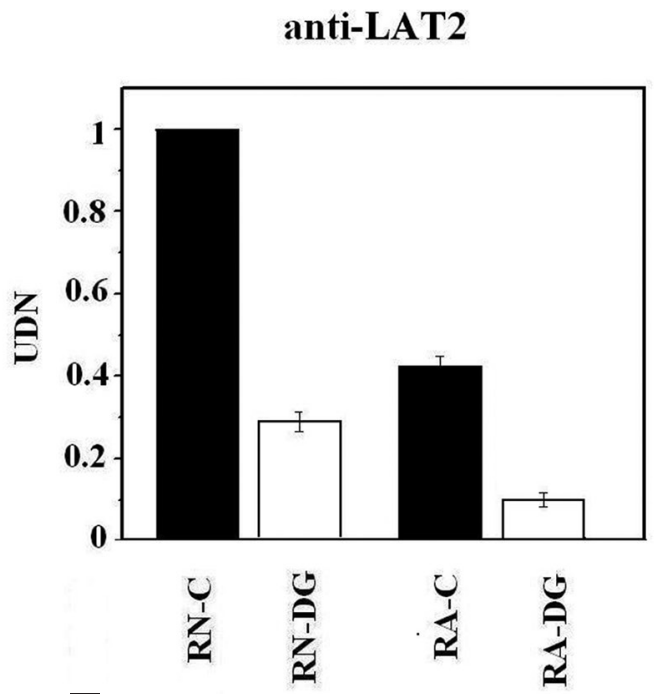

B

Figura 8. Western Blot con anti-LAT2 en los TTs aislados de músculo de ratas RN-C, RN-DG, RA-C y RA-DG; A: Las bandas corresponden a la misma cantidad en $\mathrm{mg} /$ proteína de TTs. B: Corresponde al promedio de las UDN $(n=3)$ DE. La normalización se realizó en relación a los valores de UDN máxima.

El transportador de aminoácidos SNAT2 transporta aminoácidos pequeños y neutros, como la alanina, aminoácido importante en la gluconeogénesis y la transaminación en el ME. La figura 9 A muestra la imagen representativa de la determinación de SNAT2 en las RN-C, RN-DG, RA-S control y RA-S con DG. La figura 9 B representa el análisis estadístico expresado en UDN $(n=3)$. La expresión de SNAT2 en las ratas RN-DG fue un $68 \%$ menor que en las RN-C. Sin embargo, a los 2 años se mantiene una menor expresión de SNAT2 en las RA-S con DG, siendo el $42 \%$ de las RA-S control. Similar a lo que ocurre con LAT2, la expresión de SNAT2 es muy importante en el ME del recién nacido, y esta expresión 

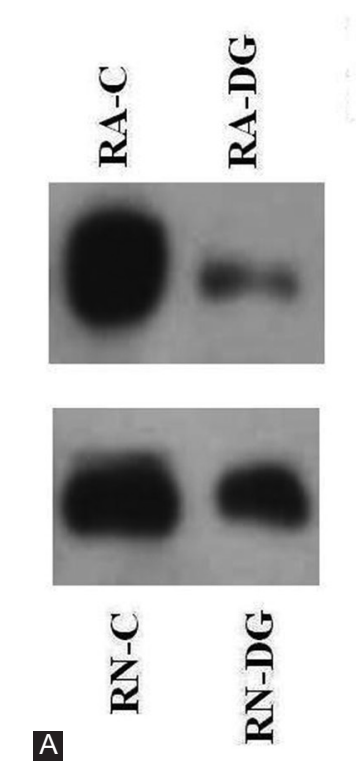

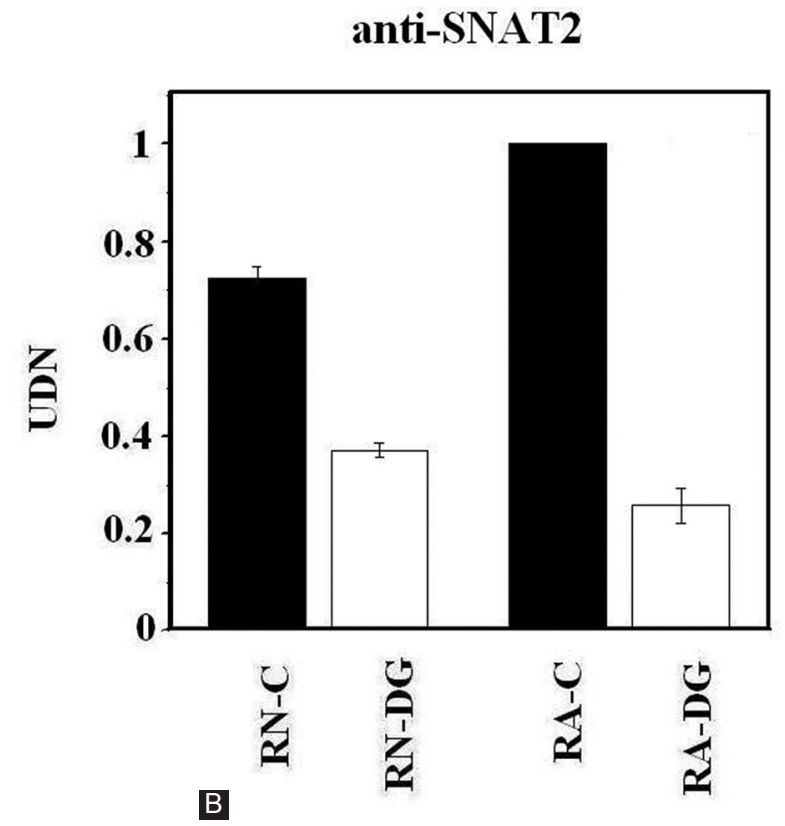

Figura 9. Western Blot con anti-SNAT2 en los TTs aislados de músculo de ratas RN-C, RN-DG, RA-S control y RA-S con DG; A: Las bandas corresponden a la misma cantidad en $\mathrm{mg} /$ proteína de TTs. B: corresponde al promedio de las UDN $(n=3) D E$. La normalización se realizó en relación a los valores de UDN máxima.

se ve disminuida en el RN-DG, efecto que se conserva hasta la vejez.

\section{Discusión}

El ME alcanza su diferenciación poco antes del nacimiento, por lo que los requerimientos energéticos y nutricionales en este periodo son fundamentales. A partir del nacimiento, el ME crece por hipertrofia y miogénesis de las células satélite ${ }^{22,23}$, pero no por división de la fibra muscular. Las alteraciones en la función muscular originadas a consecuencia de la $D G$ tienen alcances en etapas más avanzadas de la vida, aumentando el riesgo de enfermedades metabólicas, tales como la obesidad y la diabetes ${ }^{24,25}$. Se ha demostrado, en humanos y en modelos animales, que los neonatos que han sufrido DG presentan hipotrofia muscular. Sin embargo, también presentan mayor fuerza de contracción y resistencia a la fatiga desde el nacimiento y hasta la vida adulta ${ }^{16,26}$, pero no en la vejez. El paradigma que establece que la desnutrición está asociada a una función orgánica deficiente parece no seguir la regla en la función mecánica del ME rápido. Sin embargo, el cambio que se genera en el ME durante la DG tiene desventajas muy importantes en términos de su función como regulador metabólico sistémico en caso de estrés en la vida adulta.

Hemos demostrado que ratas neonatas provenientes de una DG hipocalórica desarrollan una musculatura menor, pero proporcional al tamaño del animal. Sin embargo, esta musculatura tiene características diferentes desde el punto de vista estructural y funcional. El desarrollo de los TT es significativamente menor en los músculos de las ratas con $\mathrm{DG}^{16}$.

El ME en esta condición sobreexpresa GLUT4 en las membranas de los TT, lo que podría interpretarse como una respuesta adaptativa a la restricción nutricional $^{16,27}$. Sin embargo, esta condición representa una desventaja a lo largo de la vida adulta si la ingesta de alimento en estos individuos no es adecuada en cantidad y contenido nutricional. Debido al efecto de la DG en el neonato al día 0 , el cual cursa con hipoglucemia, hipoinsulinemia y disminución en los RI en los TT, es justificado preguntarse cuál es el mecanismo que guía a los GLUT4 a sobreexpresarse en los TT del músculo de animales con DG si los RI están disminuidos.

Existe una correlación entre el aumento de los GLUT4 y la hipoglucemia; la disminución de los RI plantea que la expresión membranal de los GLUT4 no obedece a la estimulación por insulina en el ME del animal desnutrido, lo que sugiere un mecanismo diferente de señalización en el músculo control y en el de los neonatos con DG.

Todavía faltan elementos para discutir acerca del funcionamiento y el mecanismo que induce a los GLUT4 a incrementar su expresión en los TT, pero puede estar relacionado con la estimulación a través del movimiento. 


\section{Propiedades mecánicas y metabólicas del músculo esquelético}

La hipotrofia del músculo es un fenómeno que, además de observarse en la ausencia de movimiento, ocurre también como consecuencia del ayuno prolongado, en las enfermedades metabólicas descompensadas como la diabetes, las distrofias musculares y durante el proceso de envejecimiento. Adicional a la hipotrofia, existe sarcopenia en las distrofias musculares y la vejez; la pérdida de masa muscular y la disminución en la fuerza de contracción son el factor incapacitante progresivo e irreversible en el envejecimiento de los mamíferos, ya sea en estado de salud 0 enfermedad.

La sarcopenia en las ratas Wistar se inicia después del año de edad, y en los humanos sanos y físicamente activos se inicia después de los 40 años ${ }^{15,28}$. Entre los 70 y los 80 años hay una pérdida del 25 al $40 \%$ de las células del músculo, acompañada de la pérdida en la fuerza de contracción ${ }^{29}$. La disminución de la fuerza está relacionada con la disminución de la masa muscular. Sin embargo, esta disminución en la fuerza no solo obedece a esta relación física, ya que la fuerza en el músculo aislado determinada en modelos animales se calcula por unidad de masa, lo que significa que la misma masa desarrolla diferente fuerza.

Una de las explicaciones está en la composición de esta masa; la sustitución de miofibrillas por fibras de colágena y adipocitos es una observación clara y consistente con el mantenimiento de la masa muscular, pero carente de propiedades contráctiles. La complejidad de la estructura y la cantidad de proteínas involucradas en la transmisión de la fuerza, ya sea en el eje longitudinal (de tendón a tendón) o en el eje transversal (comunicación entre células musculares) a través de las proteínas costaméricas durante la contracción y la relajación, resulta en una combinación de mecanismos que explican la falla mecánica sistémica.

La disminución en el transporte de aminoácidos y los RI, la pérdida de fuerza y de regulación del calcio intracelular, así como la falta de recuperación de la fuerza después de la fatiga en la vejez, son condiciones que limitan la función del tejido más abundante en los vertebrados. El efecto de la vejez sobre la estructura y función del ME rápido se ve agravado si el organismo ha crecido en un ambiente restringido en aporte calórico durante la gestación. Esta condición limita la participación del músculo en el control sistémico de la glucemia.
La fatiga, fenómeno fisiológico intrínseco del ME que experimentan principalmente los músculos del movimiento (músculos rápidos) después de una actividad prolongada, es un indicador de la composición del tipo de fibras del músculo completo. En el caso de los músculos posturales (músculos lentos), estos experimentan poco la fatiga. Por ello, es importante estudiar el efecto del envejecimiento y de la desnutrición en músculos aislados enriquecidos en un tipo de fibras musculares.

En nuestro estudio con músculos aislados podemos investigar el fenómeno de fatiga en la vejez. Aunque la fuerza de contracción se ve seriamente afectada en el músculo rápido, la fatiga después de un tren de estimulación no se modifica. Esto indica que los mecanismos moleculares encargados de regular las concentraciones de calcio intracelular durante la contracción y la relajación no están alterados en la fracción de músculo que permanece después de la sarcopenia. Sin embargo, observamos que la actividad de la SERCA está disminuida, lo que implica un aumento en la concentración de calcio intracelular y en la expresión y la actividad de la PMCA de los TT, proteína que bombea el calcio al espacio extracelular en estado de actividad muscular prolongada, como sucede en la adaptación del músculo al ejercicio ${ }^{30}$.

En la vejez, la PMCA también se encuentra disminuida, por lo que en la actividad prolongada en la vejez cursa con un aumento del calcio citosólico y un retardo en la recuperación de la fuerza muscular si este no termina en daño permanente.

Es importante no perder de vista que el ME, además de ser un motor que genera movimiento, es una máquina metabólica que regula la concentración de glucosa plasmática, es un almacén de proteínas que abastece de aminoácidos al resto del organismo en estados de estrés prolongado y tiene propiedades sensoriales para la propiocepción.

\section{El músculo como proveedor de aminoácidos al resto de los órganos}

El transporte de aminoácidos se ve favorecido por la insulina ${ }^{31}$. En el animal con DG, el transporte de aminoácidos y los RI están disminuidos en el día 0 posnatal y en la vejez. La pérdida de la capacidad de almacenaje de proteína en el músculo no solo impone limitaciones en el crecimiento, sino que también condiciona las características de la alimentación que deberá seguir el sujeto a lo largo de la vida. 
Durante el envejecimiento, los tejidos compuestos de células que proliferan comprometen la homeostasis del músculo al responder este a las exigencias del estrés sistémico. El aporte de aminoácidos del músculo al resto de las células sugiere un aumento en la proteólisis de las proteínas estructurales. La tasa anual de pérdida muscular se ha estimado en un rango del 1 al $2 \%$ en las personas mayores de 50 años, el $25 \%$ hasta los 70 años y el $40 \%$ en las personas mayores de 80 años ${ }^{32}$.

Nuestros resultados en el modelo animal sugieren que la proteólisis del músculo como mecanismo compensatorio sistémico en la vejez, expresado como hipotrofia y sarcopenia, no es un proceso reversible, principalmente debido a la disminución en la expresión de transportadores de aminoácidos y RI en los TT.

En estas condiciones, la DG puede verse no solo como un factor deletéreo en las circunstancias presentes de demanda metabólica del animal, sino también como un acelerador del efecto de la vejez en el ME rápido.

\section{Agradecimientos}

Este trabajo ha sido financiado por la Dirección General de Apoyo al Personal Académico (DGAPA) IN218215 de la Universidad Nacional Autónoma de México (UNAM) y la Facultad de Medicina de la UNAM. Agradecemos la donación de músculo de ratas de 2 años por el laboratorio de la Dra. Mina Konigsberg de la Universidad Autónoma Metropolitana, y la colaboración de los Dres. MVZ Enrique Pinzón e Ismael Torres, de la Facultad de Medicina de la UNAM, quienes han contribuido con los modelos animales.

\section{Bibliografía}

1. Davis TA, Suryawan A, Orellana RA, et al. Postnatal ontogeny of skeletal muscle protein synthesis in pigs. J Anim Sci. 2008;86:E13-8.

2. Bryson-Richardson RJ, Currie PD. The genetics of vertebrate myogenesis. Nature Rev Genet. 2008;9:632-46.

3. Franzini-Armstrong C. Simultaneous maturation of transverse tubules and sarcoplasmic reticulum during muscle differentiation in the mouse. Developmental Biol. 1991;146:353-63.

4. Peachey LD. The sarcoplasmic reticulum and the transverse tubules of the frog's Sartorius. J Cell Biol. 1995;25:209-231.

5. Zorzano A, Palacín M, Gumà A. Mechanisms regulating GLUT4 glucose transporter expression and glucose transport in skeletal muscle. Acta Physiol Scandinav. 2005;183:43-58.
6. Hayashi T, Wojtaszewski JF, Goodyear LJ. Exercise regulation of glucose transport in skeletal muscle. Am J Physiol. 1997;273:E1039-51.

7. Mitsumoto $Y$, Klip A. Development regulation of the subcellular distribution and glycosylation of GLUT1 and GLUT4 glucose transporters during myogenesis of L6 muscle cells. J Biol Chem. 1992;267:4957-62.

8. Mitsumoto Drummond MJ, Glynn EL, Fry CS, et al. An increase in essential amino acid availability upregulates amino acid transporter expression in human skeletal muscle. Am J Physiol Edocrinol Metab. 2010;298:E1011-8.

9. Bukholder TJ. Mechanotransduction in skeletal muscle. Front Biosci. 2007;12:174-98.

10. Verdijk LB, Snijders T, Drost M, et al. Satellite cells in human skeletal muscle; from birth to old age. AGE. 2014;36:545-57.

11. Merlini L, Bonaldo P, Marzetti E. Pathophysiological mechanisms of sarcopenia in aging and in muscular dystrophy a translational approach. Front Aging Neurosci. 2015;7:153.

12. Solares-Pérez A, Álvarez $\mathrm{R}$, Crosbie $\mathrm{RH}$, et al. Altered calcium pump and secondary deficiency of gamma sarcoglycan and microspan in sarcoplasmic reticulum membranes isolated from delta sarcoglycan knockout mice. Cell Calcium. 2010;48:28-36.

13. García-Pelagio KP, Bloch RJ, Ortega A, et al. Biomechanics of the sarcolemma and costameres in single skeletal muscle fibers from normal and dystrophin-null mice. J Muscle Res Cell Motil. 2011;31:323-36.

14. Rosenberg IH. Sarcopenia: origins and clinical relevance. J Nutr. 1997;127(Suppl):990-1.

15. Sengupta $P$. The laboratory rat: relating its age with human's. Int J Prev Med. 2013;4:624-30.

16. Ramírez-Oseguera RT, Álvarez R, Pinzón-Estrada E, et al. Gestational undernourishment affects skeletal muscle mechanical properties and transverse tubule membrane structure in the new-born rat. Cell Physiol Biochem. 2013;32:1024-39.

17. Vázquez $\mathrm{P}$, Tirado-Cortés $\mathrm{A}$, Álvarez $\mathrm{R}$, et al. Reversible oxidation of vicinal-thiols motif in sarcoplasmic reticulum calcium regulatory proteins is involved in muscle fatigue mechanism. Cell Calcium. 2016;60:245-55.

18. Ortega A, Lepock JR. Use of thermal analysis to distinguish magnesium and calcium stimulated ATPase activity in isolated transverse tubules from skeletal muscle. Biochem Biophys Acta. 1995;1233:7-13.

19. Lanzeta Lanzetta PA, Álvarez LJ, Reinach PS, et al. An improved assay for nanomole amounts of inorganic phosphate. Anal Biochem. 1979;100:95-7.

20. Marzetti E, Leeuwenburgh C. Skeletal muscle apoptosis, sarcopenia and frailty at old age. Exp Gerontol. 2006;41:1234-8.

21. Denne SC, Kalhan SC. Leucine metabolism in human newborns. Am J Physiol Endocrinol. 1987;253:E608-15.

22. Bazgir B, Fathi R, Valojerdi MR, et al. Satellite cells contribution to exercise mediated muscle hypertrophy and repair. Cell J. 2017;18:473-84.

23. Dhawan J, Rando TA. Stem cells in postnatal myogenesis: molecular mechanisms of satellite cell quiescence, activation and replenishment. Trends Cell Biol. 2005;15:666-73.

24. Ozanne SE, Hales CN. The long term consequences of intrauterine protein malnutrition for glucose metabolism. Proc Nutr Soc. 1999;58:615-9.

25. George LA, Zhang L, Tuersunjiang N, et al. Early maternal undernutrition programs increased feed intake, altered glucose metabolism and insulin secretion, and liver function in aged female offspring. Am J Physiol Regul Integr Comp Physiol. 2012;302:R795-804.

26. Toscano AE, Manhñes-de-Castro R, Canon F. Effect of a low-protein diet during pregnancy on skeletal muscle mechanical properties of offspring rats. Nutrition. 2008;24:270-8.

27. Hales CN, Barker DJP. Type 2 (non-insulin-dependent) diabetes mellitus: the thrifty phenotype hypothesis. Diabetologia. 1992;35:595-601.

28. Lexell J. Human aging, muscle mass, and fiber type composition. J Gerontol A Biol Sci Med Sci. 1995;50(Spec No):11-6.

29. Kalyani RR, Corriere M, Ferrucci L. Age-related and disease-related muscle loss: the effect of diabetes, obesity, and other diseases. Lancet Diabetes Endocrinol. 2014;2:819-29.

30. Becker V, González-Serratos H, Álvarez R, et al. Effect of endurance exercise on the $\mathrm{Ca} 2+$ pumps from transverse tubule and sarcoplasmic reticulum of rabbit skeletal muscle. J Appl Physiol. 2004;97:467-74.

31. Fujita S, Rasmussen BB, Cadenas JG, et al. Effect of insulin on human skeletal muscle protein synthesis is modulated by insulin-induced changes in muscle blood flow and amino acid availability. Am J Physiol Endocrinol Metab. 2006;291:E745-54.

32. Shaw SC, Dennison EM, Cooper C. Epidemiology of sarcopenia: determinants throughout the lifecourse. Calcif Tissue Int. 2017;101:229-47. 